\title{
Magnetic Bead Separation
}

National Cancer Institute

\section{Source}

National Cancer Institute. Magnetic Bead Separation. NCI Thesaurus. Code C19304.

A cell-separation technique where magnetizable microspheres or beads are first coated with monoclonal antibody, allowed to search and bind to target cells, and are then selectively removed when passed through a magnetic field. Among other applications, the technique is commonly used to remove tumor cells from the marrow of patients who are to undergo autologous bone marrow transplantation. 